\title{
Yeast require an Intact Tryptophan Biosynthesis Pathway and Exogenous Tryptophan for Resistance to Sodium Dodecyl Sulfate
}

\author{
Laura M. Ammons ${ }^{1,2}$, Logan R. Bingham ${ }^{1,2}$, Sarah Callery ${ }^{1,2}$, Elizabeth Corley ${ }^{1,2}$, Katherine A. \\ Crowe $^{1,2}$, Jennifer K. Lipton ${ }^{1,2}$, Carlos A. Mendez ${ }^{1,2}$, Tessalyn Morrison ${ }^{1,2}$, Claudia Rallis ${ }^{1,2}$, \\ Amie J. McClellan ${ }^{2,3}$
}

\begin{abstract}
Sodium Dodecyl Sulfate, or SDS, is an anionic detergent with widespread use in industrial and household cleaning products, scientific laboratories, and personal care products such as toothpaste and shampoo. The potential toxicity of SDS has been wellcharacterized in whole organism studies and its potential effects on the environment continue to be studied. Herein, we undertake a chemical-genetic screen to explore whether low concentrations of SDS have any discernible effects at the cellular level. Our screen of the homozygous diploid yeast deletion collection identified numerous gene deletions that confer sensitivity to SDS. Subsequent bioinformatics and biological analyses reveal that yeast unable to synthesize tryptophan are especially sensitive to the presence of SDS. Interestingly, even wild-type yeast with an intact tryptophan biosynthetic pathway exhibit growth defects in the presence of SDS on media lacking tryptophan. Altogether, we have shown that low levels of SDS, primarily through effects on tryptophan availability, impact the basic cell biology of a eukaryotic cell.
\end{abstract}

Keywords: Saccharomyces cerevisiae; genome-wide chemical-genetic screen; SDS

\section{Introduction}

The anionic detergent sodium dodecyl sulfate (SDS; $\mathrm{C}_{12} \mathrm{H}_{25} \mathrm{C}_{6} \mathrm{H}_{4} \mathrm{SO}_{3} \mathrm{Na}$ ), also known as sodium lauryl sulfate, is a commonly used synthetic surfactant in industry, as well as in household cleansers and personal hygiene products including shampoo, toothpaste, and cosmetics. SDS is also extensively used in scientific laboratories, primarily to disrupt cellular or intracellular membranes, or as a protein denaturant/charge neutralizer in analytical applications such as SDS-PAGE (Polyacrylamide Gel Electrophoresis). The specific mechanism by which SDS denatures the tertiary structure of proteins is still being actively investigated (Bhuyan, 2009). Due to its widespread human use, animal studies on the effects of SDS exposure have long been conducted and studies of anionic surfactant toxicology in the environment continue to be published (Final safety report, 1983; Scott and Jones, 2000; Wibbertmann et al., 2011). Such studies have revealed that while SDS is a potent eye, skin, and respiratory tract irritant, these effects are generally temporary and alleviated by extensive rinsing and/or fresh air. Additionally, the amounts of SDS consumers are generally exposed to are orders of magnitude lower than amounts that cause adverse effects in animal studies (Wibbertmann et al., 2011). Of note, however, safety studies look broadly at toxicity by examining whole organ or system effects (visible skin irritation/dermatitis upon contact; nausea or vomiting upon ingestion, etc.).

In order to investigate whether very low concentrations of SDS have specific effects on the general biological processes of a cell, we utilized the model single-celled eukaryotic organism Saccharomyces cerevisiae, also known as the budding yeast. Yeast deletion collections exist, which permit the genome-wide analysis of growth under a given condition, for example, in the presence of a drug (Winzeler $e t$ al., 1999; Giaever et al., 2002; McClellan et al., 2007). In this study, we conducted a chemical-genetic screen examining the ability of $\sim 5,800$ homozygous diploid yeast deletion strains to grow in the absence and presence of a low concentration $(0.015 \%)$ of SDS. Our initial growth experiments, coupled with extensive re-testing and bioinformatics analyses, reveal for the first time that SDS strongly affects the viability of yeast that are unable to synthesize their own tryptophan. Further, our results suggest that an intact tryptophan biosynthesis pathway, in the absence of exogenous tryptophan, is inadequate to permit growth in the presence of SDS. Thus, it appears that SDS has specific effects on cell biological processes beyond its known abilities to disrupt cellular membranes and denature proteins.

\section{Results}

Genome-wide Screen for Nonessential Yeast Genes Required for Resistance to SDS

The entire collection of BY4743 background homozygous diploid yeast deletion strains ( $\sim 5800$ strains; Winzeler et al., 1999) was tested for growth on rich media (YPD; Yeast extract, Peptone, Dextrose) lacking or containing $0.015 \%$ SDS. This concentration of SDS was chosen based on research demonstrating that $0.01 \%$ SDS slows the growth of wild-type (WT) yeast in liquid culture (Sirisattha et al., 2004). All sets of plates were examined by at least two different individuals; deletion strains that exhibited poor growth on SDS (for example, see Figure 1) by consensus of all individuals that examined a given set of plates, were included in the initial candidate deletion strain list of 156 strains (Table 1).

1 These authors contributed equally to this publication

2 Division of Natural Sciences and Mathematics, Bennington College, Bennington, VT 05201 USA

3 Faculty Member

Corresponding Author: Amie J. McClellan, amcclellan@bennington.edu 


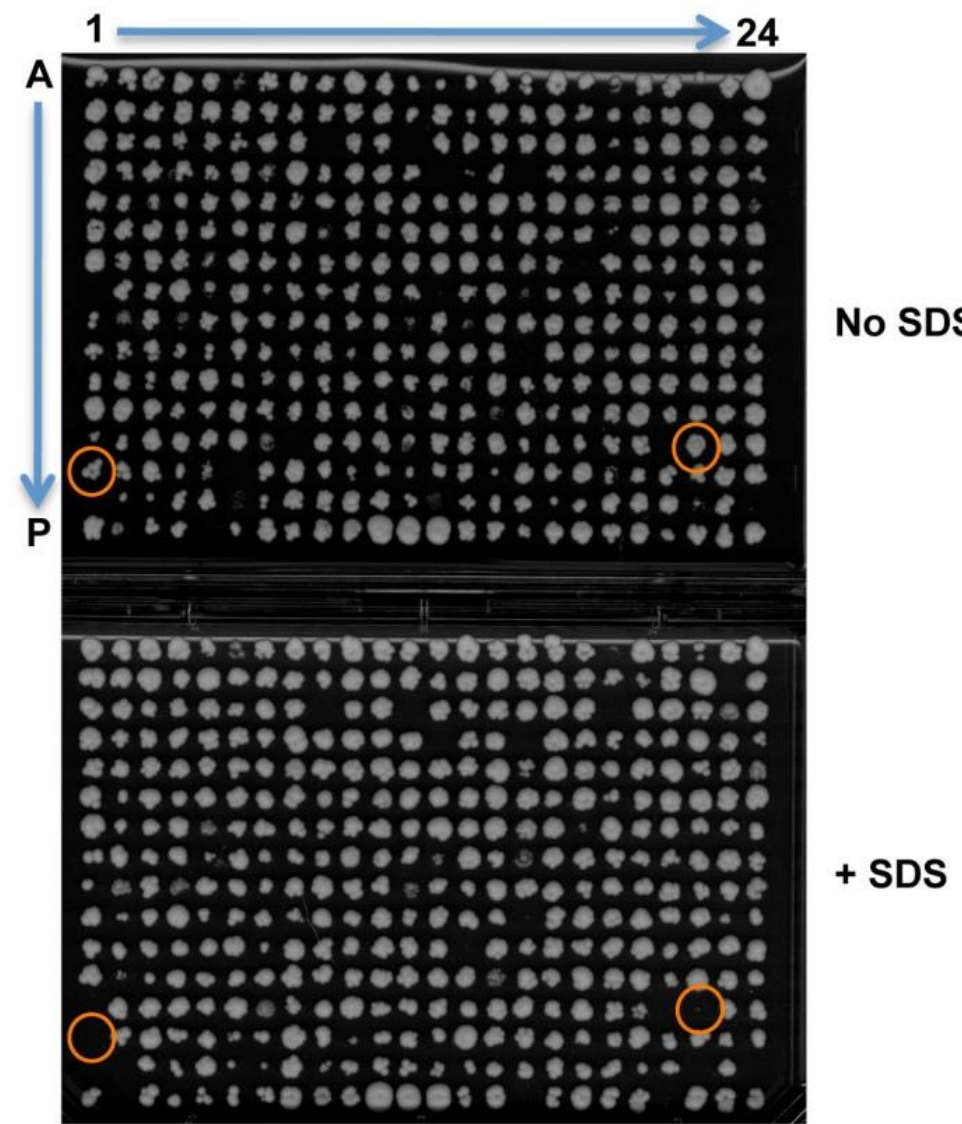

Figure 1: Example of 384-well plate spotting and growth comparison. Homozygous yeast deletion strains spotted onto rich media (YPD) without (top; No SDS) or containing (bottom; + SDS) $0.015 \%$ SDS. Orange circles indicate strains that grew in the absence, but not presence, of SDS (384-well plate coordinates M22 and N1, in this example). 
Table 1: Candidate SDS-Sensitive Yeast Deletion Strains Subjected to Validation

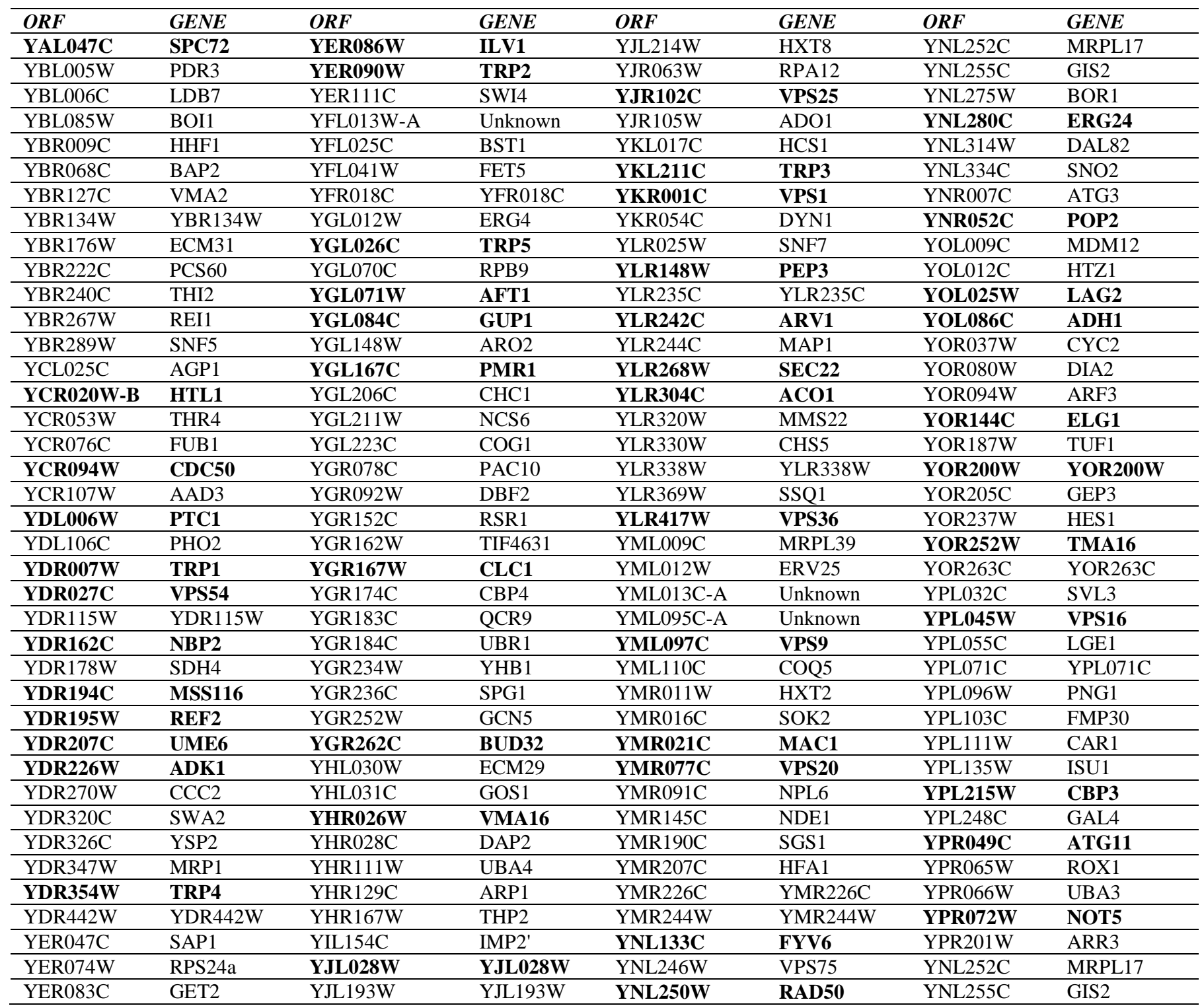

Of the $\sim 5,800$ deletion strains screened for growth on media containing $0.015 \%$ SDS, the 156 listed above were initially scored as deficient for growth in the presence of SDS. All 156 strains were subsequently individually retested by serial dilution growth assays. Those highlighted in bold above represent the 46 deletion strains ultimately confirmed to have SDS-induced growth defects (herein the data set).

Each candidate strain was then individually tested versus wild-type (WT) BY4743 yeast for growth on YPD lacking or containing SDS. Representative examples of deletion strain re-testing by serial dilution are shown in Figure 2. As expected from previous work (Sirisattha et al., 2004), some inhibition of WT growth is observed on plates containing $0.015 \%$ SDS. While many re-tested deletion strains did not exhibit a significant loss of viability on SDS (for example, Figure 2, strains $\Delta$ swi4, $\Delta$ rpal2, $\Delta$ mrpl39), nearly $30 \%$ of retested strains were confirmed to exhibit little to no growth in the presence of SDS (for example, Figure 2, strains $\Delta$ pop2, $\Delta s p c 72, \Delta$ trp3). Overall, 46 strains comprise our final data set of confirmed SDS-sensitive deletion strains (Table 1, bold). 


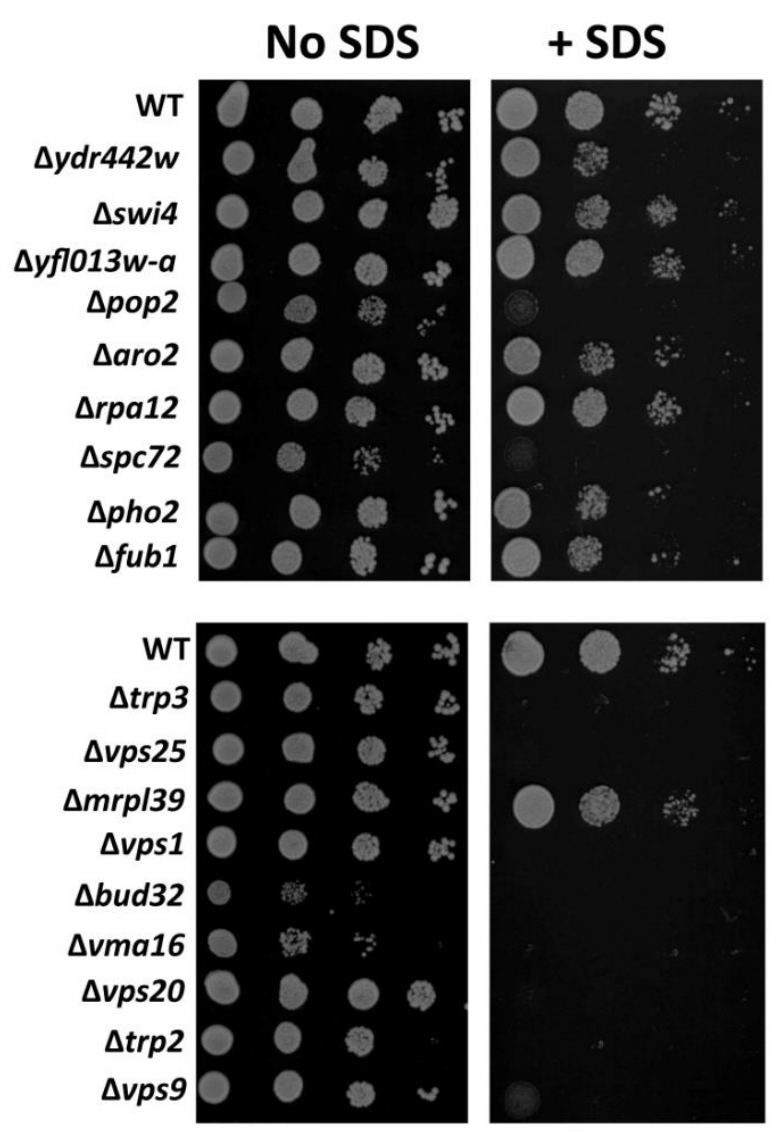

Bioinformatic Analyses Suggest Tryptophan Biosynthesis is Important for SDS Tolerance

In order to identify any specific cell biological processes in yeast affected by growth in the presence of SDS, we utilized FunSpec (http://funspec.med.utoronto.ca/; Robinson et al., 2002) to determine any statistically significant Gene Ontology (GO; Ashburner et al., 2000) classifications amongst our data set genes. Of the top five enriched GO Biological Process categories, four of them relate to amino acid biosynthesis, specifically, to the tryptophan biosynthetic pathway (Table 2; see also Figure 4). Of note, the GO classification for protein targeting to the vacuole is also statistically significant within our data set. The FunSpec analysis also identified that 24 of our 46 data set deletion strains are classified as slow growing $(\Delta s p c 72, \Delta h t l 1, \Delta v p s 54$,
Figure 2: Candidate strain re-testing. Representative serial dilutions on YPD plates lacking (No SDS) or containing (+ SDS) $0.015 \%$ SDS. Spots, from left to right, are ten-fold serial dilutions starting from cultures diluted to an $\mathrm{OD}_{600}$ of $0.01 \quad(0.01,0.001,0.0001$, $0.00001)$.
$\Delta$ mss 116, $\Delta$ ref2, $\Delta$ ume6, $\Delta a d k 1, \Delta a f t 1, \Delta$ gupl, $\Delta$ pmrl, $\Delta$ clc1,

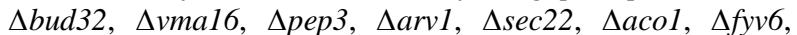
$\Delta \operatorname{rad50,\Delta erg} 24, \Delta p o p 2, \Delta a d h 1, \Delta v p s 16, \Delta$ not 5 ; hereafter collectively referred to as "slow growers"). As strains lacking those genes have inherent fitness defects (Giaever et al., 2002), it is possible that their growth will be further impeded by numerous types of drug treatments and may not be specific to SDS (see Discussion). To account for this possibility, we re-ran the FunSpec analysis with a truncated data set list excluding slow growers. Importantly, this did not change the top five enriched GO Biological Processes and, in fact, increased the statistical significance ascribed to the four categories associated with amino acid biosynthesis (Table 2, $\mathrm{p}$-value column versus p-value (slow growers removed) column). 
Table 2: Top Five Statistically Significant Gene Ontology Classifications

\begin{tabular}{|c|c|c|c|c|}
\hline $\begin{array}{l}\text { Enriched GO Biological Process } \\
\text { Categories }\end{array}$ & $\begin{array}{l}\text { Data Set Genes } \\
\text { in Category }\end{array}$ & p-value & $\begin{array}{c}\text { p-value } \\
\text { (slow growers } \\
\text { removed) }\end{array}$ & $\begin{array}{l}\text { Data Set Genes/ } \\
\text { Total Genes } \\
\text { (in Category) }\end{array}$ \\
\hline $\begin{array}{l}\text { tryptophan biosynthetic process } \\
\text { [GO:0000162] }\end{array}$ & $\begin{array}{l}\text { TRP1 TRP4 TRP2 } \\
\text { TRP5 TRP3 }\end{array}$ & $1.313 \mathrm{e}-11$ & $2.531 \mathrm{e}-13$ & $5 / 5$ \\
\hline $\begin{array}{l}\text { aromatic amino acid family } \\
\text { biosynthetic process } \\
\text { [GO:0009073] }\end{array}$ & $\begin{array}{l}\text { TRP1 TRP4 TRP2 } \\
\text { TRP5 TRP3 }\end{array}$ & $1.002 \mathrm{e}-08$ & $1.967 \mathrm{e}-10$ & $5 / 12$ \\
\hline $\begin{array}{l}\text { tryptophan metabolic process } \\
\text { [GO:0006568] }\end{array}$ & TRP1 TRP5 TRP3 & $3.165 \mathrm{e}-07$ & $3.211 \mathrm{e}-08$ & $3 / 3$ \\
\hline $\begin{array}{l}\text { protein targeting to vacuole } \\
\text { [GO:0006623] }\end{array}$ & $\begin{array}{l}\text { VPS25 VPS1 VPS9 } \\
\text { VPS9 VPS16 }\end{array}$ & $8.16 \mathrm{e}-06$ & $8.634 \mathrm{e}-06$ & $5 / 41$ \\
\hline $\begin{array}{l}\text { cellular amino acid biosynthetic } \\
\text { process [GO:0008652] }\end{array}$ & $\begin{array}{l}\text { TRP1 TRP4 ILV1 } \\
\text { TRP2 TRP5 TRP3 } \\
\end{array}$ & $5.313 \mathrm{e}-05$ & $5.643 \mathrm{e}-07$ & $6 / 98$ \\
\hline
\end{tabular}

The final data set of 46 deletion strains was analyzed by FunSpec (http://funspec.med.utoronto.ca/; Robinson et al., 2002) to identify any statistically enriched Gene Ontology (GO) Biological Process classifications. The five most significantly enriched GO Biological Processes are displayed, as well as the data set genes ascribed to each category, relevant p-values, and the fraction of total genes assigned to each category that occur in our data set.

We next analyzed our data set with regard to established connections (published genetic and/or physical interactions) between the genes therein. The resulting interaction network (Figure 3) contains 27 well-connected nodes (including 11 slow growers), six nodes with only one connection (five of the six are slow growers), and 13 loner nodes (seven of which are slow growers). The most well-connected and highconfidence module of the interaction network consists of the five genes involved in tryptophan biosynthesis, TRP1, TRP2, TRP3, TRP4, and TRP5 (Figure 4).

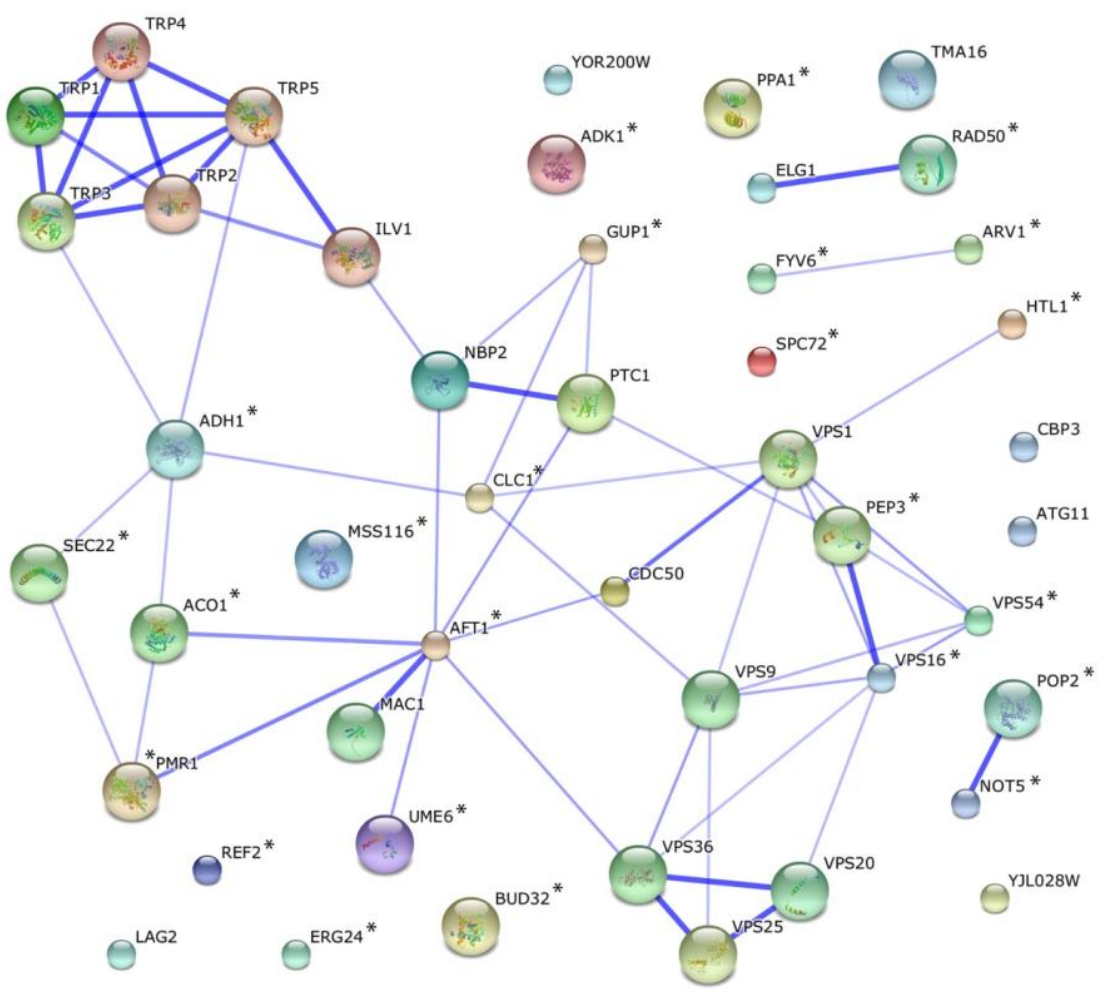

Figure 3: STRING v9.1 (Franceschini et al., 2013; http://string-db.org/) generated protein-protein interaction network for final data set. The degree of confidence for a given interaction is indicated by the thickness of the edge connecting two nodes (the thicker the edge, the more established the interaction). Inherently slow growing strains in the data set are indicated with an asterisk (*). 


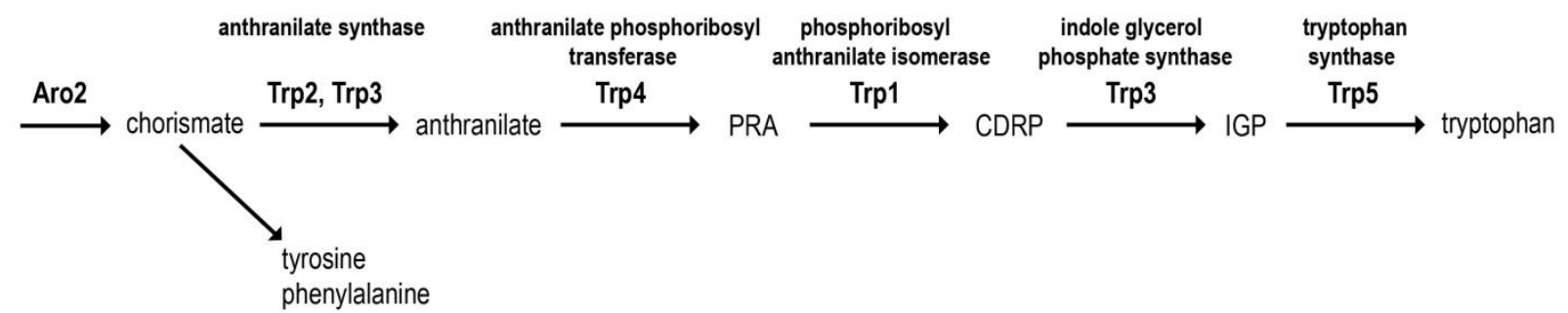

Figure 4: Outline of the yeast tryptophan biosynthesis pathway. Enzymes encoded by TRP genes are printed in bold above the relevant protein-encoding gene name(s). Abbreviations used: $\mathrm{PRA}=\mathrm{N}$-(5'-phosphoribosyl)-anthranilate, $\mathrm{CDRP}=1$ - $(\mathrm{O}-$ carboxyphenylamino)-1'-deoxyribulose-5'-phosphate, IGP = indole glycerol phosphate.

\section{The Effects of SDS on Tryptophan Biosynthesis and Uptake}

The results thus far support that yeast without the ability to make tryptophan are unable to grow in the presence of SDS. As an important control, we next tested whether restoring tryptophan biosynthesis to a deficient strain rescues growth in the presence of SDS. Indeed, $\Delta$ trpl yeast transformed with a plasmid harboring a functional TRPl gene (pESC TRP1, Agilent Technologies) grew well on YPD both lacking and containing SDS (Figure 5, bottom row). We also tested whether $A R O 2$, a gene important for the synthesis of the tryptophan, tyrosine, and phenylalanine precursor chorismate (see Figure 4), was critical for SDS tolerance. Figure 5 shows that $\triangle a r o 2$ yeast are viable both in the absence and presence of SDS, suggesting that the problem lies specifically with tryptophan synthesis and not with the general production of aromatic amino acids. Next, we explored whether it is possible that yeast lacking components of the tryptophan biosynthesis pathway cannot grow in the presence of SDS due to problems with tryptophan uptake from the media. Yeast lacking either TAT1 or TAT2, which encode a low-affinity tryptophan permease and high-affinity tryptophan permease, respectively (Schmidt et al., 1994), grew in the absence and presence of SDS (Figure 5). This result suggests that yeast with an intact tryptophan biosynthesis pathway are viable on SDS either due to synthesis of their own tryptophan, or due to adequate uptake of tryptophan from the media. To distinguish between these possibilities, we next examined the ability of WT and $\Delta t r p$ yeast to grow on YPD or synthetic media lacking tryptophan (-TRP), in the absence and presence of SDS. As expected, all strains tested grew robustly on YPD lacking SDS (Figure 6, left panel) and strains defective for tryptophan biosynthesis $(\Delta t r p 1, \Delta \operatorname{trp} 2, \Delta \operatorname{trp} 3, \Delta \operatorname{trp} 4, \Delta \operatorname{trp} 5)$ were unable to grow on YPD containing SDS, or on -TRP media lacking or containing SDS (Figure 6). Surprisingly, however, while WT yeast grew well on -TRP media lacking SDS, WT yeast grew poorly on -TRP media containing SDS, despite having an intact tryptophan biosynthetic pathway. This suggests that, at least in the presence of SDS, TRP+ yeast obtain the tryptophan that they require from the media, not via tryptophan biosynthesis.
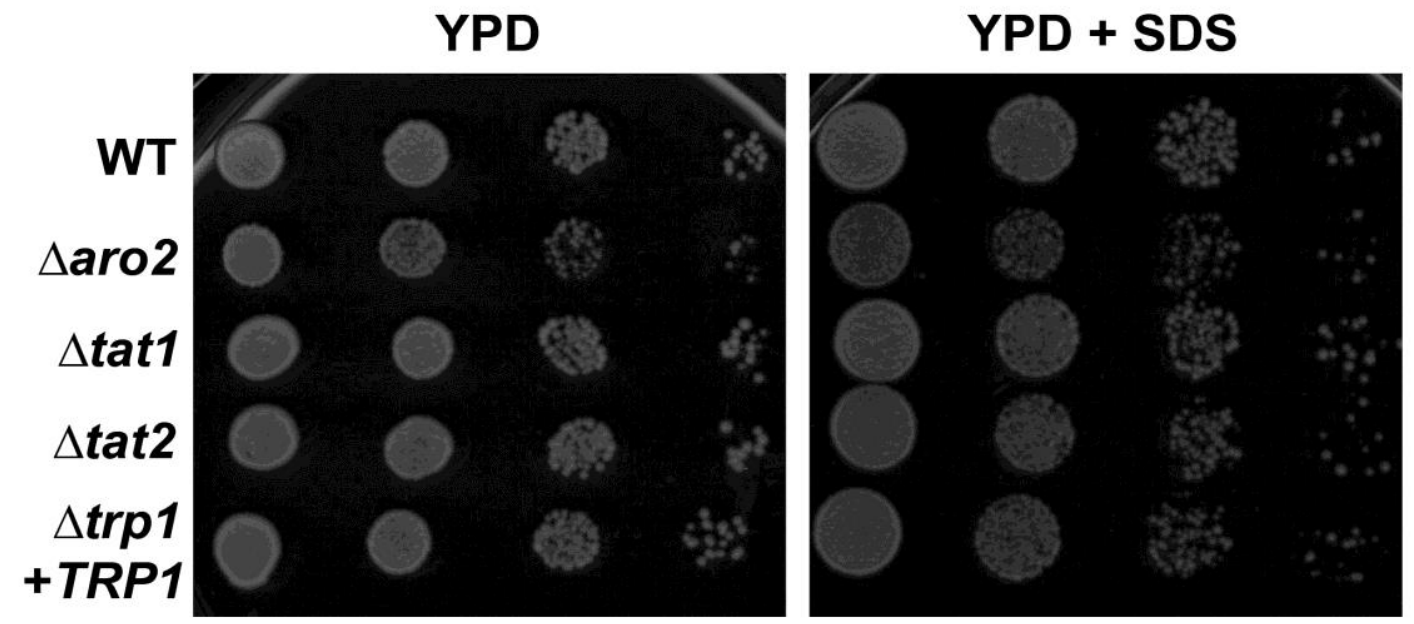

Figure 5: Yeast lacking ARO2, TAT1, or TAT2 grow on YPD containing SDS. Representative serial dilutions on YPD plates lacking or containing $0.015 \%$ SDS. Spots, from left to right, are ten-fold serial dilutions starting from cultures diluted to an $\mathrm{OD}_{600}$ of $0.01(0.01,0.001,0.0001,0.00001)$. 
YPD

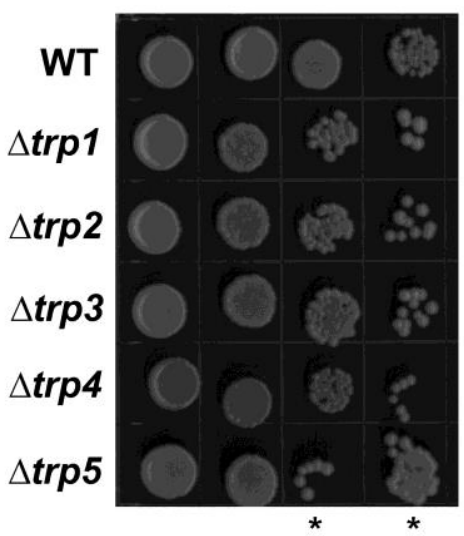

YPD + SDS

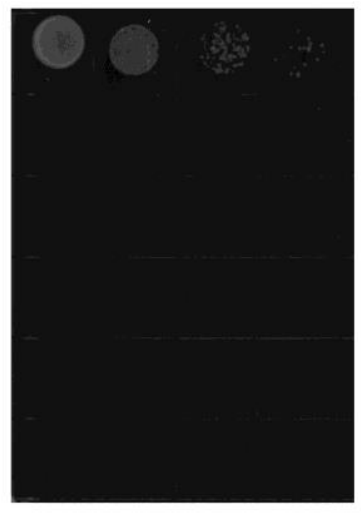

-TRP

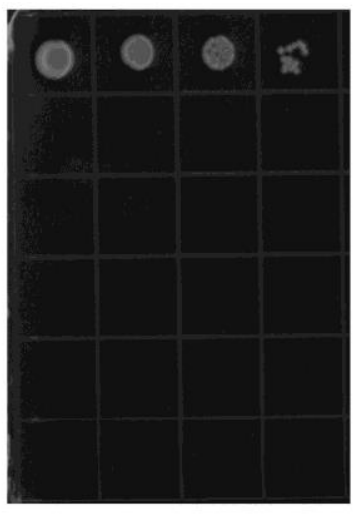

- TRP + SDS

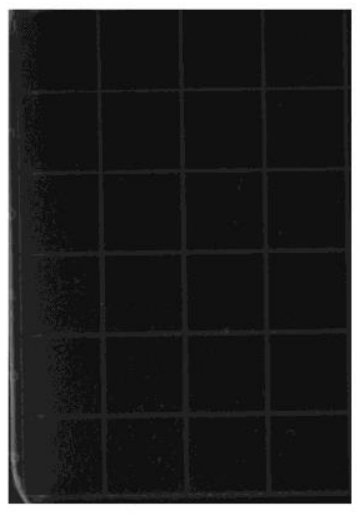

Figure 6: Representative serial dilutions on YPD and -TRP plates lacking or containing $0.015 \%$ SDS. Spots, from left to right, are ten-fold serial dilutions starting from cultures diluted to an $\mathrm{OD}_{600}$ of $0.01(0.01,0.001,0.0001,0.00001)$. Asterisks $(*)$ indicate that the 0.0001 and 0.00001 dilution spots for $\Delta$ trp5 are reversed.

\section{Discussion}

Our chemical-genetic screen of $\sim 5,800$ homozygous diploid yeast deletion strains identified 46 genes that, when deleted, compromise the ability of yeast to grow in the presence of SDS (Table 1). Bioinformatic analyses of the 46 gene data set revealed that amino acid metabolism, specifically that of tryptophan, was the primarily affected cellular process (Table 2; Figure 3). Subsequent experiments demonstrated that yeast lacking one or the other tryptophan permease (TAT1 or TAT2) grew well in the presence of SDS, suggesting either that one can compensate for the other, or that it is the process of tryptophan biosynthesis itself that is directly affected by SDS. Notably, while WT yeast grow in the presence of SDS on rich media (YPD), they are unable to grow well in the presence of SDS on media lacking tryptophan (-TRP; Figure 6). Thus, we conclude that yeast that are unable to synthesize their own tryptophan, as well as yeast with intact tryptophan biosynthetic pathways but no available exogenous tryptophan, exhibit SDS-induced growth defects.

A significant number of yeast deletion strains in our final data set are known slow growers (Giaever et al., 2002). As mentioned briefly in the Results, yeast deletion strains known to have fitness defects are often further growth-impaired in the presence of various chemicals and, therefore, their occurrence in our data set may not be specific to an effect of SDS on growth, but rather reflect a larger issue of drug tolerance and sensitivity in these yeast. As examples, consider two slow growers from our data set: $\Delta a r v 1$ and $\Delta a f t 1$ yeast; $\Delta a r v 1$ yeast not only grow slowly in the absence of perturbation, but exhibit decreased resistance to numerous chemicals with various modes of action, including the protein synthesis inhibitor cycloheximide (Alamgir et al., 2010; Kapitzky et al., 2010), the DNA-damaging antibiotic bleomycin (Kapitsky et al., 2010), the antifungal nystatin (Tinkelenberg et al., 2008), and the HMG-CoA reductase inhibitor lovastatin (Fei W. et al., 2008). Yeast lacking AFT1 also display decreased resistance to multiple chemicals, including various alcohols (ethanol, methanol, propan-1-ol; Auesukaree et al., 2009), the anti-malarial drug quinine (Dos Santos and Sa-Correia, 2011), and the DNA modifying drug methyl methanesulfonate (Svensson et al., 2011). Now, consider those same two genes in the context of our data set interaction network (Figure 3). While $A R V 1$ has only a single connection, and that is to another known slow grower ( $F Y V 6)$, the AFT1 node has eight network connections and is connected by two different pathways to the primary TRP gene cluster. Additionally, like $\Delta a f t 1$ yeast, $\Delta \operatorname{trp} 1, \Delta \operatorname{trp} 2, \Delta \operatorname{trp} 3$, $\Delta \operatorname{trp} 4$, and $\Delta \operatorname{trp} 5$ yeast are hypersensitive to growth in the presence of ethanol (Yoskikawa et al., 2009), and yeast lacking TRP1, TRP2, or TRP5 grow poorly in the presence of quinine (Khozoie et al., 2009). Altogether, this makes it tempting to speculate that $A F T 1$ may truly belong as part of our analysis, while the exclusion of $A R V 1$ is unlikely to change our findings. Overall, it seems prudent to carefully consider that some slow growing deletion strains with promiscuous chemical sensitivities are likely specifically affected by SDS, and, as such, their inclusion in our data set may be informative as far as further elucidating the specific biological effects of SDS on yeast.

In addition to the specific examples of ethanol and quinine given above, tryptophan metabolism is often observed as a drug-sensitive process in yeast. In some instances, there is good evidence to support a mechanism, for example, the very strong structural similarity between tryptophan and quinine suggests competition for permease binding as a likely point of action (Khozoie et al., 2009). We are unable at this time to provide a concrete explanation for our observed effects of SDS on tryptophan metabolic pathways in yeast. However, it is known that the overexpression of TAT2 or TRP1 rescues the sensitivity of yeast to FK506, an immunosuppressive drug (Heitman et al., 1993; Schmidt et al., 1994), as well as ameliorating other conditions that impair tryptophan uptake in yeast. As our results suggest that both tryptophan uptake and, perhaps more critically, tryptophan biosynthesis, are important for resistance to SDS, similar overexpression experiments would be helpful in further clarifying the underlying mechanism(s) at work. Additionally, as there is some functional redundancy between TAT1 and TAT2 as tryptophan permeases, it would be interesting to test the effect of SDS on yeast lacking both genes. Since the double deletion of both TAT1 and TAT2 is not lethal (Schmidt et al., 1994), these experiments are viable next steps to extend our findings. Finally, a more complete picture of the mode of action of SDS on yeast cellular 
processes may be obtained by conducting this screen on the available heterozygous yeast deletion collection. This would permit the addition to our data set of any essential genes involved by identifying genes that are haploinsufficient in the presence of SDS. This study thus represents the first step toward characterizing the mode of action of SDS at the level of the individual cell and its biological processes.

\section{Experimental Procedures}

Yeast Media Preparation and Yeast Transformation

Media were prepared and yeast transformation was conducted following standard procedures (Adams et al., 2007).

Solid Media Screen of Homozygous Diploid Yeast Deletion Collection

The yeast homozygous diploid collection, comprising $\sim 5,800$ deletion strains (Winzeler et al., 1999; Thermo Scientific) was arrayed using a Floating Pin Replicator (V\&P Scientific, Inc.) onto solid YPD media (1\% yeast extract, $2 \%$ peptone, $2 \%$ glucose) lacking or containing $0.015 \%$ SDS (Sigma-Aldrich). Plates were incubated at $30{ }^{\circ} \mathrm{C}$ until colonies appeared (generally 2-3 days). Plates were inspected by eye to identify deletion strains that grew poorly in the presence, but not absence, of SDS.

\section{Bioinformatic Analyses}

The final data set of 46 deletion strains was analyzed using FunSpec (Robinson et al., 2002; http://funspec.med.utoronto.ca/) to identify statistically enriched Gene Ontology Biological Process classifications. All available databases were selected for the query, the Bonferroni correction was not utilized, and the p-value cutoff was set to 0.001 . The final data set of 46 deletion strains was also analyzed using STRING v9.1 (Franceschini et al., 2013; http://string-db.org/). The resulting protein-protein interaction network is shown in Figure 3.

\section{Serial Dilution Growth Assays}

WT (BY4743; Thermo Scientific) and candidate SDSsensitive deletion strains were inoculated into $2 \mathrm{~mL}$ YPD and grown overnight in a shaking incubator at $30{ }^{\circ} \mathrm{C}$. The next day, the optical density at $600 \mathrm{~nm}\left(\mathrm{OD}_{600}\right)$ of each strain was determined with a GENESYS ${ }^{\text {TM }} 20$ visible spectrophotometer (Thermo Scientific). Each strain was then diluted to an $\mathrm{OD}_{600}$ of $0.01,0.001,0.0001$, and 0.0001 and $5 \square \mathrm{L}$ of each dilution was spotted onto solid media plates. Plates were incubated at $30{ }^{\circ} \mathrm{C}$ until colonies appeared (generally 2-3 days). For experiments comparing growth on media containing or lacking tryptophan, both YPD and -TRP dropout media (2\% glucose, $0.68 \%$ yeast nitrogen base lacking amino acids, and $0.2 \%$ amino acid dropout mix lacking tryptophan) were utilized.

\section{Acknowledgements}

The authors thank Gerry Bolgos and David Norman for reagent ordering, yeast media preparation, yeast transformations, and general laboratory maintenance. A. J. McClellan acknowledges the generous assistance and support of Tim Stearns and Anna Ballew O'Connell, upon whose undergraduate research course development work at Stanford University this project was modeled. The authors thank the Bennington College Genetics students of Spring 2007, Spring 2008, and Spring 2011, who collectively laid the groundwork for the successful execution of this project by the Fall 2013 Genetics class.

\section{References}

Abe, F. and H. Iida. 2003. Pressure-Induced Differential Regulation of the Two Tryptophan Permeases Tat1 and Tat 2 by Ubiquitin Ligase Rsp5 and Its Binding Proteins, Bul1 and Bul2. Molecular and Cellular Biology 23: 75667584. doi: 10.1128/MCB.23.21.7566-7584.2003

Adams, A., D. E. Gottschling, C. A. Kaiser, and T. Stearns. 1997. Methods in Yeast Genetics (Cold Spring Harbor, NY: Cold Spring Harbor Laboratory Press).

Alamgir M., V. Erukova, M. Jessulat, A. Azizi, A. Golshani. 2010. Chemical-genetic profile analysis of five inhibitory compounds in yeast. BMC Chemical Biology 10. doi: 10.1186/1472-6769-10-6

Ashburner M., C. A. Ball, J. A. Blake, D. Botstein, H. Butler, J. M. Cherry, A. P. Davis, K. Dolinski, S. S. Dwight, J. T. Eppig, M. A. Harris, D. P. Hill, L. Issel-Tarver, A. Kasarskis, S. Lewis, J. C. Matese, J. E. Richardson, M. Ringwald, G. M.Rubin, and G.Sherlock. 2000. Gene ontology: tool for the unification of biology. Nature Genetics 25: 25-29. doi:10.1038/75556

Auesukaree C., A. Damnernsawad, M. Kruatrachue, P. Pokethitiyook, C. Boonchird, Y.Kaneko, S. Harashima. 2009. Genome-wide identification of genes involved in tolerance to various environmental stresses in Saccharomyces cerevisiae. Journal of Applied Genetics 50: 301-310. doi: 10.1007/BF03195688

Bhuyan, A. K. 2010. On the mechanism of SDS-induced protein denaturation. Biopolymers. 93: 186-199. DOI: 10.1002/bip.21318

Dos Santos, S. C. and I. Sá-Correia. 2011. A genome-wide screen identifies yeast genes required for protection against or enhanced cytotoxicity of the antimalarial drug quinine. Molecular Genetics and Genomics. 286: 333-346. doi: 10.1007/s00438-011-0649-5

Fei W., G. Alfaro, B-P. Muthusamy, Z. Klaassen, T. R. Graham, H. Yang and C. T. Beh. 2008. Genome-Wide Analysis of Sterol-Lipid Storage and Trafficking in Saccharomyces cerevisiae. Eukaryotic Cell 7: 401-414. doi: 10.1128/EC.00386-07

Final report on the safety assessment of sodium lauryl sulfate and ammonium lauryl sulfate. 1983. International Journal of Toxicology, Volume 2: 127-181. doi: $10.3109 / 10915818309142005$.

Franceschini, A., D. Szklarczyk, S. Frankild, M. Kuhn, M. Simonovic, A. Roth, J. Lin, P. Minguez, P. Bork, C. von Mering, and L. J. Jensen. 2013. STRING v9.1: proteinprotein interaction networks, with increased coverage and integration. Nucleic Acids Research 41: D808-15. doi: 10.1093:nar:gks1094

Fujita K, A. Matsuyama, Y. Kobayashi, H. Iwahashi. 2006. The genome-wide screening of yeast deletion mutants to identify the genes required for tolerance to ethanol and other alcohols. FEMS Yeast Research. 6: 744-750. doi:10.1111/j.1567-1364.2006.00040.x 
Giaever G., A. M. Chu, L. Ni, C. Connelly, L. Riles, S. Véronneau, S. Dow, A. Lucau-Danila, K. Anderson, B. André, A. P. Arkin, A. Astromoff, M. El-Bakkoury, R. Bangham, R. Benito, S. Brachat, S. Campanaro, M. Curtiss, K. Davis, A. Deutschbauer, K. D. Entian, P. Flaherty, F. Foury, D. J. Garfinkel, M. Gerstein, D. Gotte, U. Güldener, J. H. Hegemann, S. Hempel, Z. Herman, D. F. Jaramillo, D. E. Kelly, S. L. Kelly, P. Kötter, D. LaBonte, D. C. Lamb, N. Lan, H. Liang, H. Liao, L. Liu, C. Luo, M. Lussier, R. Mao, P. Menard, S.L. Ooi, J. L. Revuelta, C. J. Roberts, M. Rose, P. Ross-Macdonald, B. Scherens, G. Schimmack, B. Shafer, D. D. Shoemaker, S. Sookhai-Mahadeo, R. K. Storms, J. N. Strathern, G. Valle, M. Voet, G. Volckaert, C. Y. Wang, T. R. Ward, J. Wilhelmy, E. A. Winzeler, Y. Yang, G. Yen, E. Youngman, K. Yu, H. Bussey, J. D. Boeke, M. Snyder, P. Philippsen, R. W. Davis, and M. Johnston. 2002. Functional profiling of the Saccharomyces cerevisiae genome. Nature 418: 387-391. doi:10.1038/nature00935

Heitman, J., A. Koller, J. Kunz, R. Henriquez, A. Schmidt, N. R. Movva, and M. N. Hall. 1993. The immunosuppressant FK506 inhibits amino acid import in Saccharomyces cerevisiae. Molecular and Cellular Biology 13: 50105019.

http://www.ncbi.nlm.nih.gov/pmc/articles/PMC360149/pdf /molcellb00020-0566.pdf

Kapitzky L., P. Beltrao, T. J. Berens, N. Gassner, C. Zhou, A. Wüster, J. Wu, M. M. Babu, S. J. Elledge, D. Toczyski, R. S. Lokey, N. J. Krogan. 2010. Cross-species chemogenomic profiling reveals evolutionarily conserved drug mode of action. Molecular Systems Biology 6 . doi: $10.1038 / \mathrm{msb} .2010 .107$

Khozoie, C., R. J. Pleass, and S. V. Avery. 2009. The Antimalarial Drug Quinine Disrupts Tat2p-mediated Tryptophan Transport and Causes Tryptophan Starvation. Journal of Biological Chemistry 284: 17968-17974. doi: 10.1074/jbc.M109.005843

McClellan, A. J., Y. Xia, A. M. Deutschbauer, R. W. Davis, M. Gerstein, and J. Frydman. 2007. Diverse cellular functions of the Hsp90 molecular chaperone uncovered using systems approaches. Cell 131: 121-35. http://dx.doi.org/10.1016/j.cell.2007.07.036

Robinson, M. D., J. Grigull, N. Mohammad, and T. R. Hughes. 2002. FunSpec: a web-based cluster interpreter for yeast. BMC Bioinformatics 3. doi: 10.1186/1471-21053-35

Schmidt, A., M. N. Hall, and A. Koller. 1994. Two FK506 Resistance-Conferring Genes in Saccharomyces cerevisiae, TAT1 and TAT2, Encode Amino Acid Permeases Mediating Tyrosine and Tryptophan Uptake. Molecular and
Cellular Biology 14: 6597-6606. doi: 10.1128/MCB.14.10.6597

Scott, M. J. and M. N. Jones. 2000. The biodegradation of surfactants in the environment. Biochimica et Biophysica Acta 1508: 235-251. http://dx.doi.org/10.1016/S03044157(00)00013-7

Sirisattha, S., Y. Momose, E. Kitagawa, and H. Iwahashi. 2004. Toxicity of anionic detergents determined by Saccharomyces cerevisiae microarray analysis. Water Research 38: 61-70. http://dx.doi.org/10.1016/j.watres.2003.08.027

Svensson, J. P., L. Quirós Pesudo, R. C. Fry, Y. A. Adeleye, P. Carmichael, and L. D. Samson. 2011. Genomic phenotyping of the essential and non-essential yeast genome detects novel pathways for alkylation resistance. BMC Systems Biology 5. doi: 10.1186/1752-0509-5-157

Tinkelenberg, A. H., Y. Liu, F. Alcantara, S. Khan, Z. Guo, M. Bard, and S. L. Sturley. 2000. Mutations in Yeast ARV1 Alter Intracellular Sterol Distribution and Are Complemented by Human ARV1. Journal of Biological Chemistry 275: 40667-40670. doi: 10.1074/jbc.C000710200

Wibbertmann A, I. Mangelsdorf, K. Gamon, R. Sedlak. 2011 Toxicological properties and risk assessment of the anionic surfactants category: Alkyl sulfates, primary alkane sulfonates, and $\alpha$-olefin sulfonates. Ecotoxicology and Environmental Safety 74: 1089-1106. http://dx.doi.org/10.1016/j.ecoenv.2011.02.007

Winzeler E. A., D. D. Shoemaker, A. Astromoff, H. Liang, K. Anderson, B. Andre, R. Bangham, R. Benito, J. D. Boeke, H. Bussey, A. M. Chu, C. Connelly, K. Davis, F. Dietrich, S. W. Dow, M. El Bakkoury, F. Foury, S. H. Friend, E. Gentalen, G. Giaever, J. H. Hegemann, T. Jones, M. Laub, H. Liao, N. Liebundguth, D. J. Lockhart, A. LucauDanila, M. Lussier, N. M'Rabet, P. Menard, M. Mittmann, C. Pai, C. Rebischung, J. L. Revuelta, L. Riles, C. J. Roberts, P. Ross-MacDonald, B. Scherens, M. Snyder, Sookhai-S. Mahadeo, R. K. Storms, S. Véronneau, M. Voet, G. Volckaert, T. R. Ward, R. Wysocki, G. S. Yen, K. Yu, K. Zimmermann, P. Philippsen, M. Johnston, R. W. Davis. 1999. Functional characterization of the Saccharomyces cerevisiae genome by gene deletion and parallel analysis. Science 285: 901-906. doi:10.1126/science.285.5429.901

Yoshikawa K., T. Tanaka, C. Furusawa, K. Nagahisa, T. Hirasawa, and H. Shimizu. 2009. Comprehensive phenotypic analysis for identification of genes affecting growth under ethanol stress in Saccharomyces cerevisiae. FEMS Yeast Research 9: 32-44. doi: 10.1111/j.15671364.2008.00456 